\title{
Effect of anticoagulants and antiplatelet agents on the efficacy of intravesical BCG treatment of bladder cancer: A systematic review
}

\author{
Nader Fahmy, MD, PhD, FRCSC,; Alejandro Lazo-Langner, MD, MSc; ${ }^{\dagger}$ Alla E. lansavichene, BSc, MLIS, Ste- \\ phen E. Pautler, BSC, MD, FRCSC*
}

*Divisions of Urology \& Surgical Oncology, Departments of Surgery \& Oncology, Schulich School of Medicine \& Dentistry, Western University, London, ON; †Department of Medicine, Division of Hematology; and Department of Epidemiology and Biostatistics; and Department of Oncology, Western University, London ON; §library Services, London Health Sciences Centre, London ON

Cite as: Can Urol Assoc J 2013;7 (11-12):e740-9. http://dx.doi.org/10.5489/cuaj.1213

Published online November 8, 2013.

\section{Abstract}

We performed a systematic review of publications describing a correlation between oral anticoagulant medications and intravesical BCG outcome. We collected information on the impact of such medications on tumour recurrence and progression and we excluded papers not reporting outcome correlations. Patients were divided into group 1 and 2 based on whether they were taking or not taking any anticoagulant medications. A total of 7 manuscripts published between 1990 and 2009 were included in this study. Data heterogeneity precluded meta-analysis. In studies combining all anticoagulant medications, 3 out of $5(60 \%)$ publications did not identify any difference in outcome, while $2(40 \%)$ documented significantly more recurrences in group 1 patients. In studies performing multivariate analysis and only examining the intake of 1 medication, warfarin alone seemed to be associated with increased risk of bladder tumour recurrences and progression following intravesical BCG treatment, while ASA alone seemed to be associated with more protective effects. There is no strong evidence to support the allegations of a protective role of ASA and a deleterious role for warfarin. Further, well-designed experimental and clinical studies are needed to clarify the mechanism of action of intravesical BCG along with possible drug interactions.

\section{Introduction}

Bladder cancer is the sixth most common non-cutaneous cancer and the ninth most common cause of overall cancer death in Canada. ${ }^{1}$ Eighty percent of urothelial cancers present as non-invasive disease. Transurethral resection of bladder tumours (TURBT), along with intravesical Bacillus Calmette-Guérin (BCG) management, is presently considered the most effective management for intermediate and high-risk non-invasive tumours. Despite a significant reduc- tion in recurrence and progression rates following BCG, still up to $40 \%$ of patients will not respond and up to $50 \%$ may even progress. ${ }^{2,3}$ Consequently, studies have attempted to define factors that may influence BCG success.

The precise mechanism of BCG antitumour response remains undefined. BCG binding to the extracellular matrix was documented to be a key step in the initiation of its antitumour response. ${ }^{4,5}$ In experimental studies, medications that inhibit fibrin clot formation have significantly decreased its antitumour activity, ${ }^{4}$ while antifibrinolytic medications improved its outcome. ${ }^{6,7}$

A concern regarding the efficacy of intravesical BCG administration thus emerges when taking into account that urothelial cancers are more common in older patients who often have a significant history of vascular diseases and are frequently taking systemic anticoagulants or antiplatelet agents. Multiple publications have tested the outcome of BCG in patients receiving anticoagulant medications with variable results.

The goal of the current study was to perform a systematic review of the literature to determine if administering intravesical BCG in patients on oral anticoagulant or antiplatelet medications affects the prognosis.

\section{Methods}

A systematic review was performed in conformity to the Preferred Reporting Items for Systematic Reviews and Metaanalysis (PRISMA) statement. ${ }^{8}$

\section{Finding relevant studies}

We systematically searched MEDLINE (1946 to January 2013) using both Ovid SP and PubMed search interfaces, EMBASE (1974 to Week 1, 2013), BIOSIS Previews (January 2013), Web of Science with Conference Proceedings (January 2013), and the Cochrane Central Register of Controlled Trials 
(January 2013) databases; we looked for studies assessing the effect of anticoagulants and antiplatelet agents on the efficacy of BCG intravesical therapy in patients with bladder cancer. We used various combinations of subject headings and text words, such as BCG vaccine, Bacillus CalmetteGuérin, anticoagulants, antiplatelet, antiaggregant, platelet aggregation inhibitors, blood platelets, platelet aggregation, warfarin, acetylsalicylic acid, clopidogrel, fibrin clot inhibitor, urinary bladder neoplasms, transitional cell carcinoma (TCC), urothelial cell carcinoma (UCC), bladder cancer, bladder neoplasm, and bladder tumour.

The search strategies were modified for each electronic database using database-specific search terms, field names and syntax. In addition, the bibliographies of relevant publications, internet-based registry of clinical trials (clinicaltrials.gov), Health Technology Assessment (HTA) agencies, Google and Google Scholar were also searched to identify additional citations and ongoing trials. We excluded papers in languages other than English.

\section{Criteria for considering articles for review}

Studies were included if they met the following criteria: (1) the article involves patients with bladder tumours managed with an intravesical BCG instillation; (2) the study entails a cohort of patients who are on at least one oral anticoagulant or antiplatelet medication; and (3) the manuscript included information on prognosis.

For publications studying the outcome of different management modalities, if shown, only data concerning outcome of intravesical BCG instillation was included. Since the goal of this study was to assess association of oral anticoagulant or antiplatelet medication in BCG treated patients with prognosis, all papers not reporting any outcome correlations were excluded. Manuscripts involving anticoagulant medications administered by routes other than orally (e.g. intravesically) were also excluded.

Relevant data were extracted into predesigned custommade spreadsheets. If available, the outcomes related to a single distinct anticoagulant or antiplatelet medications were extracted separately. Studies were assessed for inclusion by 1 reviewer and corroborated by 2 other independent reviewers. Differences were resolved by consensus.

For descriptive purposes, the cohort of patients taking an anticoagulant or antiplatelet medication was labelled group 1 and those not on an anticoagulant or antiplatelet medication were called group 2 .

The primary endpoint was tumour recurrence. The secondary endpoints were (1) recurrence stage and/or grade; (2) tumour progression; and (3) disease-free interval (time to recurrence or progression).

\section{Statistical analysis}

A formal meta-analysis was not conducted due to the heterogeneity amongst the publications regarding the reported study populations, oral anticoagulants or antiplatelet drugs used, tumour pathologies, BCG regimens and outcomes. Data were extracted and summarized using central tendency measures and ranges as provided by the authors.

\section{Results}

\section{Search results and characteristics of included studies}

We identified 375 citations; after removing duplicate citations, 311 records were assessed for relevance. In total, 303 records were excluded. One pertinent record that was only published as a conference abstract was also excluded. ${ }^{9}$ In total, 7 studies met the inclusion criteria (Fig. 1) and were included in this analysis. Four (57\%) of these studies were North American, ${ }^{10-13} 2$ (29\%) Australian ${ }^{14,15}$ and one (14\%) European. ${ }^{16}$ These papers described investigations conducted between 1981 and 2006 and were published between 1990 and 2009. For all manuscripts, the outcome analysis in relation to fibrin clot inhibitors was computed retrospectively. The included studies featured a total of 1423 patients with primary or recurrent bladder urothelial carcinoma treated with intravesical BCG. None of the publications included patients with upper tract urothelial carcinoma. They involved 341 patients on at least 1 anticoagulant or antiplatelet medication (Group 1).

Baseline patient characteristics and study endpoints for all studies are shown in Table 1; medication history, tumour characteristics and BCG information are in Table 2.

While 5 studies ${ }^{10,13-16}$ combined the outcome of different medications taken either separately or together (Table 3 ), only 3 articles ${ }^{10-12}$ tested the outcome of only 1 type of anticoagulant or antiplatelet medication (Table 3, Table 4). All manuscripts reported tumour recurrence following intravesical BCG. Tumour progression was evaluated in 3 studies. ${ }^{10,11,13}$ Multivariate analyses was only performed in 3 studies $^{10-12}$ (Table 3, Table 4).

Effects of anticoagulant or antiplatelet medications on tumor recurrence

\section{Studies combining patients on multiple anticoagulant or antiplatelet medica- tions taken either separately or together}

Two studies ${ }^{14,15}$ documented significantly higher overall tumour recurrence rates in Group 1. One study ${ }^{16}$ showed a non-significant tendency towards more recurrences in Group 2 patients, while 2 other manuscripts did not show differences among both groups ${ }^{10,13}$ even after controlling for 


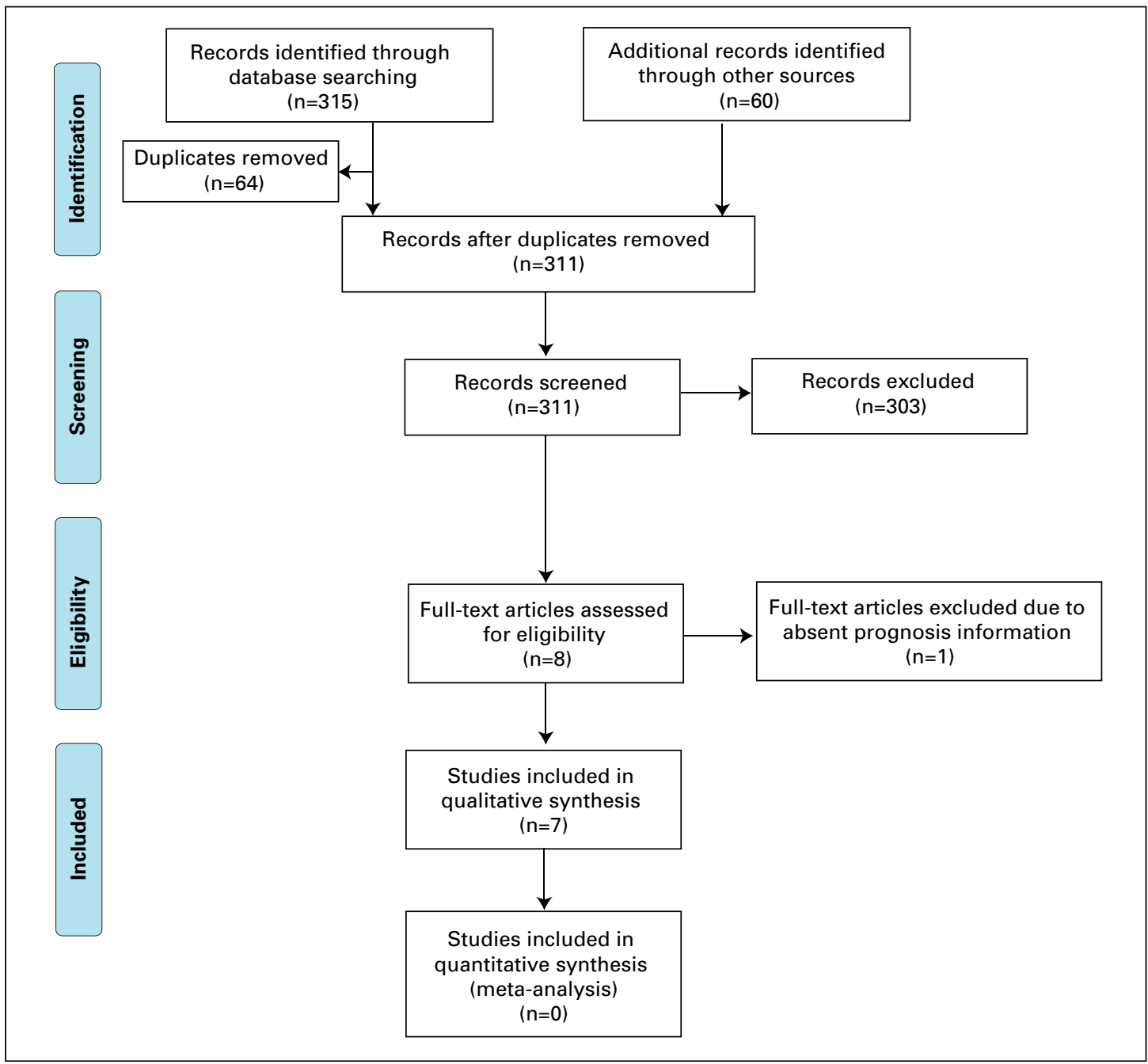

Fig. 1. PRISMA Study Selection Flow Diagram. Adapted from Moher et al. ${ }^{8}$

the type of anticoagulant or antiplatelet medication used. ${ }^{10}$ When excluding tumour progression from overall recurrences, 1 of the 2 latter studies ${ }^{13}$ revealed a significantly increased rate of superficial tumour recurrences in Group 1 patients. Time to recurrence was assessed in 2 studies $^{10,16}$ and was not significantly different between both groups (Table 3 ).

\section{Studies assessing patients taking only one type of anticoagulant or anti- platelet medication}

For patients taking oral warfarin alone, 1 study did not document any difference in recurrence and in time to recurrence among both groups by univariate analysis..$^{10}$ In 2 studies using multivariate analyses, warfarin intake alone was associated with significantly increased risks of recurrences in Group 1 patients following intravesical BCG treatment. ${ }^{10,12}$ In patients taking only systemic acetylsalicylic acid (ASA), by means of univariate analysis, 1 study reported significantly higher risk of tumour recurrence in Group 2 patients, ${ }^{11}$ whereas another study did not. ${ }^{8}$ When using multivariate analyses, 1 study documented evidence of significantly decreased risk of recurrence within Group 1 patients after controlling for age, sex, smoking status, maintenance BCG, and the presence of carcinoma in situ (CIS) or papillary tumour. ${ }^{11}$ Another study however failed to show any significant difference in recurrence rates among both groups after controlling for age, initial tumour stage (superficial vs. invasive), and anticoagulation medication used..$^{10}$

Effects of anticoagulant or antiplatelet medications on tumour progression

\section{Studies combining patients on multiple anticoagulant or antiplatelet medica- tions taken either separately or together}

There was neither a significant difference in tumour progression nor in time to progression in both groups when tested by univariate analyses. ${ }^{10,13}$ 


\begin{tabular}{|c|c|c|c|c|c|c|c|}
\hline & $\begin{array}{c}\text { Hudson et al. }{ }^{13} \\
1990\end{array}$ & $\begin{array}{c}\text { P'ng et al. }^{15} \\
1993\end{array}$ & $\begin{array}{c}\text { Witjes et al. }{ }^{16} \\
1993\end{array}$ & $\begin{array}{c}\text { Rogerson }^{14} \\
1994\end{array}$ & $\begin{array}{c}\text { Heiner et al. }{ }^{12} \\
2008\end{array}$ & $\begin{array}{l}\text { Gee et al. }{ }^{11} \\
2008\end{array}$ & $\begin{array}{c}\text { Boorjian et al. }{ }^{10} \\
2009\end{array}$ \\
\hline $\begin{array}{l}\text { Study years } \\
\text { included }\end{array}$ & $\begin{array}{l}\text { Dec 1981-June } \\
1989\end{array}$ & $1985-1990$ & $\begin{array}{l}\text { Apr 1987-Dec } \\
1991\end{array}$ & $\begin{array}{l}\text { Dec 1987-Oct } \\
1991\end{array}$ & $\begin{array}{l}\text { Dec 1999-July } \\
2004\end{array}$ & $\begin{array}{l}\text { June 1991-Sep } \\
2003\end{array}$ & $\begin{array}{l}\text { July 1978-Nov } \\
2006\end{array}$ \\
\hline Study type & Retrospective & Retrospective & Retrospective & Retrospective & Retrospective & Retrospective & Retrospective \\
\hline Institution & $\begin{array}{l}\text { - Washington } \\
\text { university } \\
\text { - Jewish } \\
\text { hospital of St } \\
\text { Louis }\end{array}$ & $\begin{array}{l}\text { - Royal } \\
\text { Brisbane } \\
\text { Hospital } \\
\text { - Princess } \\
\text { Alexandra } \\
\text { Hospital }\end{array}$ & $\begin{array}{l}\text { - Dutch } \\
\text { Southeast } \\
\text { Cooperative } \\
\text { Urological } \\
\text { Group }\end{array}$ & $\begin{array}{l}\text { Heidelberg } \\
\text { Repatriation } \\
\text { Hospital }\end{array}$ & $\begin{array}{l}\text { - Augusta } \\
\text { Veterans Affairs } \\
\text { Medical Center } \\
\text { - Medical } \\
\text { College of } \\
\text { Georgia }\end{array}$ & $\begin{array}{l}\text { - University } \\
\text { of Wisconsin } \\
\text { Hospital and } \\
\text { Clinics }\end{array}$ & $\begin{array}{l}\text { - Memorial } \\
\text { Sloan Kettering } \\
\text { Cancer Center }\end{array}$ \\
\hline Province/country & Missouri, USA & $\begin{array}{l}\text { Queensland, } \\
\text { Australia }\end{array}$ & Netherlands & $\begin{array}{l}\text { Victoria, } \\
\text { Australia }\end{array}$ & Georgia, USA & $\begin{array}{l}\text { Wisconsin, } \\
\text { USA }\end{array}$ & New York, USA \\
\hline Total patients & 162 & 45 & 313 & 56 & 58 & 154 & 1218 \\
\hline Included patients & 149 & 45 & 183 & 38 & 58 & 43 & 907 \\
\hline $\begin{array}{l}\text { Inclusion } \\
\text { selection criteria }\end{array}$ & $\begin{array}{l}\text { Available } \\
\text { medication } \\
\text { record }\end{array}$ & $\begin{array}{l}\text { All patients } \\
\text { included }\end{array}$ & $\mathrm{N} / \mathrm{A}$ & $\begin{array}{l}\text { Available } \\
\text { medication } \\
\text { record }\end{array}$ & $\mathrm{N} / \mathrm{A}$ & $\begin{array}{l}\text { Available } \\
\text { records }\end{array}$ & $\begin{array}{l}\text { - Available } \\
\text { records, } \\
\text { - No prior } \\
\text { surgical } \\
\text { intervention } \\
\text { - >1990 }\end{array}$ \\
\hline $\begin{array}{l}\text { Anticoagulant } \\
\text { G1 }\end{array}$ & 29 & 9 & 42 & 13 & 7 & 20 & 221 \\
\hline Control G2 & 120 & 36 & 141 & 25 & 51 & 23 & 686 \\
\hline $\begin{array}{l}\text { Male:Female } \\
\text { ratio } \\
\text { Statistical } \\
\text { significance }\end{array}$ & $\mathrm{N} / \mathrm{A}$ & $33: 12$ & $\mathrm{~N} / \mathrm{A}$ & $\mathrm{N} / \mathrm{A}$ & $\mathrm{N} / \mathrm{A}$ & $\begin{array}{l}36: 7 \\
\text { More males in } \\
\text { G1 (NS) }\end{array}$ & $\begin{array}{l}721: 186 \\
\text { More males } \\
\text { in } \mathrm{G} 1 \\
(p<0.01)\end{array}$ \\
\hline $\begin{array}{l}\text { Age, years } \\
\text { (range) } \\
\text { Statistical } \\
\text { significance }\end{array}$ & $\mathrm{N} / \mathrm{A}$ & $\begin{array}{l}\text { Mean both } \\
\text { groups: } 69 \\
(36-92)\end{array}$ & $\mathrm{N} / \mathrm{A}$ & $\mathrm{N} / \mathrm{A}$ & $\mathrm{N} / \mathrm{A}$ & $\begin{array}{l}\text { Mean } \\
-\mathrm{G} 1: 72 \\
-\mathrm{G} 2: 63 \\
p<0.01\end{array}$ & $\begin{array}{l}\text { Median } \\
-\mathrm{G} 1: 69 \\
-\mathrm{G} 2: 65 \\
p<0.01\end{array}$ \\
\hline $\begin{array}{l}\text { Follow-up } \\
\text { (range) }\end{array}$ & $\begin{array}{l}\text { Mean } \\
- \text { G1: } 26.5 \\
\text { months } \\
\text { - G2: } 29.8 \\
\text { months }\end{array}$ & $\begin{array}{l}\text { Mean both } \\
\text { groups } \\
20.3 \text { months } \\
\text { Median both } \\
\text { groups } \\
16 \text { months } \\
(3-56)\end{array}$ & $\begin{array}{l}\text { Mean } \\
- \text { G1: } 22.5 \\
\text { months (4.4- } \\
\text { 49.5) } \\
\text { - G2: } 20.8 \\
\text { months (2.6- } \\
53.2 \text { ) }\end{array}$ & $\begin{array}{l}\text { Mean varied } \\
\text { among stages } \\
\text { ranging } \\
\text { from } 6 \text { to } 28 \\
\text { months } \\
(2-36)\end{array}$ & N/A & N/A & $\begin{array}{l}\text { Median } \\
4.2 \text { years in } \\
\text { patients without } \\
\text { recurrence } \\
\text { and } 3.7 \text { years } \\
\text { without } \\
\text { progression }\end{array}$ \\
\hline $\begin{array}{l}\text { Primary } \\
\text { endpoint studied }\end{array}$ & $\begin{array}{l}\text { Tumour } \\
\text { recurrence }\end{array}$ & $\begin{array}{l}\text { Persistent or } \\
\text { recurrence } \\
\text { tumour at } \\
\text { first follow-up } \\
\text { in } 3 \text { months }\end{array}$ & $\begin{array}{l}\text { Disease } \\
\text { (recurrence) } \\
\text { free interval }\end{array}$ & $\begin{array}{l}\text { Persistent or } \\
\text { recurrence } \\
\text { disease at } 6 \\
\text { weeks }\end{array}$ & $\begin{array}{l}\text { Response } \\
\text { to therapy } \\
\text { categorized in } \\
\text { relation to age } \\
\text { - 1: (complete) } \\
\text { - 2: (recurrence } \\
\text { at increase } \\
\text { interval or dec. } \\
\text { grade/stage } \\
\text { - 3: (recurrence } \\
\text { same interval } \\
\text { or same stage/ } \\
\text { grade) } \\
\text { - 4: (recurrence } \\
\text { shorter interval } \\
\text { or higher stage/ } \\
\text { grade) }\end{array}$ & $\begin{array}{l}\text { Recurrence- } \\
\text { free and } \\
\text { progression- } \\
\text { free intervals }\end{array}$ & $\begin{array}{l}\text { Tumour } \\
\text { recurrence and } \\
\text { progression to } \\
\text { open surgery }\end{array}$ \\
\hline
\end{tabular}




\begin{tabular}{|c|c|c|c|c|c|c|c|c|}
\hline & & $\begin{array}{c}\text { Hudson et al. }{ }^{13} \\
1990\end{array}$ & $\begin{array}{c}\text { P'ng et al. }{ }^{15} \\
1993\end{array}$ & $\begin{array}{c}\text { Witjes et al. }{ }^{16} \\
1993\end{array}$ & $\begin{array}{c}\text { Rogerson }^{14} \\
1994\end{array}$ & $\begin{array}{l}\text { Heiner et al. }{ }^{12} \\
2008\end{array}$ & $\begin{array}{c}\text { Gee et al. }{ }^{11} \\
2008\end{array}$ & $\begin{array}{c}\text { Boorjian et al. }{ }^{10} \\
2009\end{array}$ \\
\hline & Gp1/Gp2 & $29 / 120$ & $9 / 36$ & $42 / 141$ & $13 / 25$ & $7 / 51$ & $20 / 23$ & $221 / 686$ \\
\hline \multirow[t]{2}{*}{ 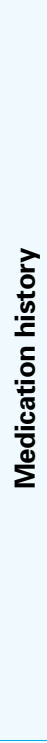 } & $\begin{array}{l}\text { Anticoagulant } \\
\text { medication } \\
\text { used in G1 }\end{array}$ & $\begin{array}{l}\text { - Warfarin } \\
(n=1) \\
\text { - ASA (n=21) } \\
\text { - Dipyridamole } \\
(n=7) \\
\text { - Indomethacin } \\
\text { ( } n=4) \\
\text { - Ibuprofen } \\
(n=3)\end{array}$ & $\begin{array}{l}\text { - Warfarin } \\
(n=1) \\
\text { - ASA }(n=4) \\
\text { - NSAID }(n=4)\end{array}$ & $\begin{array}{l}\text { - Warfarin } \\
\text { ( } n=15) \\
\text { - ASA ( } n=18) \\
\text { - Dipyridamole } \\
\text { (n=4) } \\
\text { - Indomethacin } \\
\text { (n=2) } \\
\text { - Naproxen } \\
(n=1) \\
\text { - Ibuprofen } \\
(n=3) \\
\text { - Diclofenac } \\
\text { (n=4) }\end{array}$ & $\begin{array}{l}\text { - NSAIDs } \\
(n=13)\end{array}$ & $\begin{array}{l}\text { - Warfarin } \\
(n=7)\end{array}$ & ASA $(n=20)$ & $\begin{array}{l}\text { - Warfarin } \\
(n=52) \\
\text { - ASA ( } n=170) \\
\text { - Clopidogrel } \\
(n=34)\end{array}$ \\
\hline & $\begin{array}{l}\text { Other } \\
\text { concomitant } \\
\text { medications }\end{array}$ & $\begin{array}{l}\text { Antibiotics, } \\
\text { CVS, } \\
\text { respiratory, } \\
\text { DM, CNS, } \\
\text { GIT, steroids, } \\
\text { thyroid, } \\
\text { vitamins, } \\
\text { antihistaminics }\end{array}$ & $\begin{array}{l}\text { Unknown, } \\
\text { No } \\
\text { Antibiotics }\end{array}$ & $\begin{array}{l}\text { Antibiotics } \\
\text { (more } \\
\text { significant in } \\
\text { G1), CVS, CNS, } \\
\text { respiratory, } \\
\text { paracetamol } \\
\text { and others }\end{array}$ & N/A & $\mathrm{N} / \mathrm{A}$ & $\mathrm{N} / \mathrm{A}$ & $\mathrm{N} / \mathrm{A}$ \\
\hline \multirow{3}{*}{ 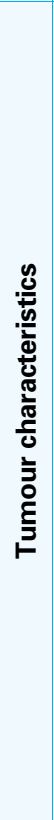 } & $\begin{array}{l}\text { Primary/ } \\
\text { recurrent } \\
\text { urothelial } \\
\text { carcinoma }\end{array}$ & $\begin{array}{l}\text { Primary or } \\
\text { recurrent }\end{array}$ & $\mathrm{N} / \mathrm{A}$ & $\begin{array}{l}\text { Primary or } \\
\text { recurrent }\end{array}$ & $\begin{array}{l}\text { Primary or } \\
\text { recurrent }\end{array}$ & $\mathrm{N} / \mathrm{A}$ & $\mathrm{N} / \mathrm{A}$ & $\mathrm{N} / \mathrm{A}$ \\
\hline & pT stage & $\begin{array}{l}\text { CIS, pTa, pT1, } \\
\text { G1: } 24 \%, 38 \% \text {, } \\
38 \% \\
\text { G2: } 22 \%, 42 \% \text {, } \\
36 \% \\
\text { - No stats }\end{array}$ & $\begin{array}{l}\text { CIS, pTa, } \\
\text { (not stratified } \\
\text { by anticoag. } \\
\text { gps) }\end{array}$ & $\begin{array}{l}\text { CIS, pTa, pT1 } \\
\text { G1: } 9 \%, 60 \% \text {, } \\
31 \% \\
\text { G2: } 11 \%, 64 \% \text {, } \\
25 \% \\
\text { - No stats }\end{array}$ & $\begin{array}{l}\text { CIS, pTa, } \\
\text { pT1,p T4 (not } \\
\text { stratified by } \\
\text { anticoag. } \\
\text { group) } \\
\text { [1 pt had } \\
\text { history of pT2, } \\
\text { treated with } \\
\text { ERT and rec } \\
\text { as T1) }\end{array}$ & $\begin{array}{l}\text { CIS , pTa, } \\
\text { pT1 (not } \\
\text { stratified } \\
\text { by anticoag } \\
\text { group) }\end{array}$ & $\begin{array}{l}\text { CIS, pure } \\
\text { papillary } \\
\text { bladder cancer } \\
\text { G1: } 75 \%, 40 \% \\
\text { G2: } 91 \%, 13 \% \\
\text { - Non- } \\
\text { significant }\end{array}$ & $\begin{array}{l}\text { Stages } \\
\text { imputed as: } \\
\text { - [pTa, pT1, } \\
\text { CIS] } \\
\text { - [pT2, pT3] } \\
\text { - Unknown } \\
\text { G1: } 69 \%, 2 \% \text {, } \\
29 \% \\
\text { G2: 70\%, 5\%, } \\
26 \% \\
\text { - No stats }\end{array}$ \\
\hline & Grade & $\begin{array}{l}\text { Grade I, II, III } \\
\text { G1: } 17 \%, 69 \% \text {, } \\
14 \% \\
\text { G2: } 21 \%, 63 \% \text {, } \\
16 \%\end{array}$ & $\begin{array}{l}\text { Grade I, } \\
\text { II, III (not } \\
\text { stratified by } \\
\text { anticoagulant } \\
\text { groups) }\end{array}$ & $\mathrm{N} / \mathrm{A}$ & N/A & $\mathrm{N} / \mathrm{A}$ & $\mathrm{N} / \mathrm{A}$ & $\begin{array}{l}\text { Grades } \\
\text { imputed as: } \\
\text { Low, high and } \\
\text { unknown } \\
\text { G1: } 9 \%, 50 \% \text {, } \\
42 \% \\
\text { G2: } 13 \%, 48 \% \text {, } \\
39 \%\end{array}$ \\
\hline
\end{tabular}

Studies assessing patients taking only one type of anticoagulant or antiplatelet medication

Among patients taking oral warfarin alone, using both univariate and multivariate analyses, 1 study indicated significantly higher progression risk in Group 1 patients with a shorter median time to progression..$^{10}$ In patients taking only systemic ASA, using univariate analyses, 1 study indicated no difference in tumour progression among both groups, ${ }^{11}$ while another study documented significantly decreased risk of progression among Group 1 patients. ${ }^{10}$ On multivariate analysis, only 1 study reported a significantly decreased risk of progression to open surgery following intravesical BCG instillation in patients taking ASA. ${ }^{10}$

\section{Discussion}

Along with TURBT, intravesical BCG has become a common treatment to manage intermediate- and high-risk noninvasive bladder cancer. Intravesical BCG was shown to 


\begin{tabular}{|c|c|c|c|c|c|c|c|c|}
\hline & & $\begin{array}{c}\text { Hudson et al. }{ }^{13} \\
1990\end{array}$ & $\begin{array}{c}\text { P'ng et al. }{ }^{15} \\
1993\end{array}$ & $\begin{array}{c}\text { Witjes et al. }{ }^{16} \\
1993\end{array}$ & $\begin{array}{c}\text { Rogerson }^{14} \\
1994\end{array}$ & $\begin{array}{l}\text { Heiner et al. }{ }^{12} \\
2008\end{array}$ & $\begin{array}{l}\text { Gee et al. }{ }^{11} \\
2008\end{array}$ & $\begin{array}{c}\text { Boorjian et al. }{ }^{10} \\
2009\end{array}$ \\
\hline \multirow{6}{*}{ 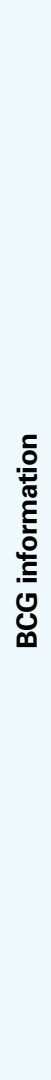 } & BCG strain & Pasteur & Pasteur strain & $\begin{array}{l}\text { TICE* }(n=25) \\
\text { and RIVM } \\
(n=17)\end{array}$ & Pasteur strain & TICE* & N/A & $\mathrm{N} / \mathrm{A}$ \\
\hline & $\begin{array}{l}\text { Dose } \\
\text { Dilution } \\
\text { Time in } \\
\text { Bladder }\end{array}$ & $\begin{array}{l}120 \mathrm{mg} \\
\text { in } 50 \mathrm{cc} \\
\text { normal saline } \\
2 \text { hours }\end{array}$ & $\begin{array}{l}120 \mathrm{mg} \\
\text { in N/A } \\
\text { N/A }\end{array}$ & $\begin{array}{l}5 \times 10^{8} \\
\text { in } 50 \mathrm{cc} \\
\text { normal saline } \\
\mathrm{N} / \mathrm{A}\end{array}$ & $\begin{array}{l}60 \mathrm{mg} \text { or } 120 \\
\mathrm{mg} \\
\text { in } 60 \mathrm{cc} \text { normal } \\
\text { saline } \\
1.5 \text { hours }\end{array}$ & $\begin{array}{l}50 \mathrm{mg} \\
\text { in } 50 \mathrm{cc} \\
\text { normal saline } \\
\mathrm{N} / \mathrm{A}\end{array}$ & $\begin{array}{l}\text { N/A } \\
N / A \\
N / A\end{array}$ & $\begin{array}{l}N / A \\
N / A \\
N / A\end{array}$ \\
\hline & $\begin{array}{l}\text { Interval given } \\
\text { post-TURBT }\end{array}$ & $\begin{array}{l}\text { within } 6 \\
\text { months }\end{array}$ & $\mathrm{N} / \mathrm{A}$ & 7 to $15 \mathrm{~d}$ & N/A & $\mathrm{N} / \mathrm{A}$ & $\mathrm{N} / \mathrm{A}$ & $\mathrm{N} / \mathrm{A}$ \\
\hline & $\begin{array}{l}\text { Course } \\
\text { induction } \\
\text { cycle }\end{array}$ & $\begin{array}{l}\text { - } 45 \text { had } 6 \text { or } \\
12 \text { week initial } \\
\text { BCG course } \\
-104 \text { had } 1 \text { or } \\
\text { more } 6 \text { week } \\
\text { BCG courses }\end{array}$ & $\begin{array}{l}1 / \text { week } \times 6 \\
\text { weeks }\end{array}$ & $\begin{array}{l}-1 / \text { week } \times 6 \\
\text { weeks } \\
-14 \% \text { G11 and } \\
17 \% \text { G2 had a } \\
\text { second } 6 \text {-week } \\
\text { course }\end{array}$ & $\begin{array}{l}-1 / \text { week } \times 6 \\
\text { weeks } \\
- \text { Some } \\
\text { patients } \\
\text { received a } \\
\text { second course }\end{array}$ & $\begin{array}{l}\text { 1/week × } 6 \\
\text { weeks }\end{array}$ & $\mathrm{N} / \mathrm{A}$ & $\begin{array}{l}\text { 1/week × } 6 \\
\text { weeks }\end{array}$ \\
\hline & $\begin{array}{l}\text { Maintenance } \\
\text { BCG }\end{array}$ & N/A & N/A & $N / A$ & N/A & $\begin{array}{l}\text { - If no } \\
\text { progression } \\
\text {->3 weekly } \\
\text { treatments } \\
\text { for } 3 \text { years } \\
\text { - Some } \\
\text { patients } \\
\text { recruited } \\
\text { while on } \\
\text { maintenance }\end{array}$ & $\begin{array}{l}\text { Not all patients } \\
\text { G1: } 3(15 \%) \\
\text { G2: } 6(26 \%)\end{array}$ & $\mathrm{N} / \mathrm{A}$ \\
\hline & $\begin{array}{l}\text { BCG } \\
\text { complications }\end{array}$ & N/A & $\mathrm{N} / \mathrm{A}$ & $\begin{array}{l}\text { Bacterial } \\
\text { cystitis G1>G2 }\end{array}$ & $\begin{array}{l}\text { Not } \\
\text { stratified by } \\
\text { anticoagulant } \\
\text { group }\end{array}$ & $\begin{array}{l}-\mathrm{G} 1: 1 / 7 \\
(14.3 \%) \\
-\mathrm{G} 2: 4 / 17 \\
(36.2 \%)\end{array}$ & $\mathrm{N} / \mathrm{A}$ & $\mathrm{N} / \mathrm{A}$ \\
\hline
\end{tabular}

significantly decrease tumour recurrence and progression when compared to TURBT alone. Still, up to $40 \%$ of patients receiving intravesical BCG failed to respond and as high as $50 \%$ of patients even progressed. ${ }^{2,3}$ Thus, it becomes intuitively evident to try and examine potential risks influencing BCG treatment success.

The exact mechanism by which BCG creates its response remains unclear. It may be achieved through several mechanisms. ${ }^{17}$ TURBT initially exposes the lamina propria and basement membrane of the urothelium leading to fibrin clot formation, altogether rich in fibronectin. The live attenuated strain of Mycobacterium bovis can thus adhere to the newly exposed fibronectin and undergo endocytosis resulting into a massive release of cytokines, chemokines and leads to activation of natural killer cells and cytotoxic T lymphocytes. ${ }^{18}$ This BCG-fibronectin binding capacity directly correlates with BCG anti-tumor activity. ${ }^{19}$ This key role of fibronectin in initiating an antitumour response was documented in a murine model, when administering anti-fibronectin antibodies lead to a total loss of BCG anti-tumour activity. ${ }^{4}$ Likewise, intravesical heparin administration inhibited
BCG-fibronectin binding in a rabbit model. ${ }^{7}$ Conversely, antifibrinolytic agents given intravenously ${ }^{6}$ or intravesically ${ }^{7}$ significantly improved BCG antitumour responses in experimental models. It was thus postulated that medications inhibiting fibrin clot formation would adversely influence BCG efficacy. Based on these findings, antifibrinolytic agents were administered intravesically (to prevent their systemic adverse effects) in a randomized prospective double blinded controlled study including 257 non-invasive low- to intermediate-risk bladder cancer patients. This study reported significantly favourable outcomes on a short median followup of 2 years. ${ }^{20}$

Since bladder tumours are more common in older patients who frequently suffer from cardiac and/or cerebrovascular diseases and are often dependent on anticoagulant or antiplatelet medications, the efficacy of intravesical BCG in these patients has been questioned. Therefore, we systematically reviewed the literature to try and identify if concomitant intake of anticoagulant/antiplatelet medications affects the prognosis of intravesical BCG. For descriptive analyses, patients were divided into Groups 1 and 2 based 
Table 3. Studies combining the outcome of different anticoagulant or antiplatelet medications

\begin{tabular}{|c|c|c|c|c|c|c|c|}
\hline & $\begin{array}{c}\text { Hudson et al. }{ }^{13} \\
1990\end{array}$ & $\begin{array}{c}\text { P'ng et al. }^{15} \\
1993\end{array}$ & $\begin{array}{l}\text { Witjes et } \\
\text { al. }{ }^{16} 1993\end{array}$ & $\begin{array}{c}\text { Rogerson }^{14} \\
1994\end{array}$ & $\begin{array}{c}\text { Heiner et al. }{ }^{12} \\
2008\end{array}$ & Gee et al. ${ }^{11} 2008$ & $\begin{array}{c}\text { Boorjian et al. }{ }^{10} \\
2009\end{array}$ \\
\hline $\begin{array}{c}\text { G1/G2 } \\
\text { Medication }\end{array}$ & $\begin{array}{c}29 / 120 \\
\text { Combined }\end{array}$ & $\begin{array}{c}9 / 36 \\
\text { Combined }\end{array}$ & $\begin{array}{c}42 / 141 \\
\text { Combined }\end{array}$ & $\begin{array}{c}13 / 25 \\
\text { Combined }\end{array}$ & $\begin{array}{c}7 / 51 \\
\text { Warfarin }\end{array}$ & $\begin{array}{c}20 / 23 \\
\text { ASA }\end{array}$ & $\begin{array}{c}221 / 686 \\
\text { Combined }\end{array}$ \\
\hline $\begin{array}{l}\text { - Recurrence in } \\
\text { G1 }\end{array}$ & $-15 / 29(52 \%)^{*}$ & - $8 / 9(88.9 \%)$ & $\begin{array}{l}-13 / 42 \\
(31 \%)^{*}\end{array}$ & $\begin{array}{l}-11 / 13 \\
(84.6 \%)^{*}\end{array}$ & $-N / A$ & $-8 / 20(40 \%)^{*}$ & $\begin{array}{l}-189 / 221 \\
(85.5 \%)^{*}\end{array}$ \\
\hline $\begin{array}{l}\text { - Time to } \\
\text { recurrence } \\
\text { (range) }\end{array}$ & $-N / A$ & - NA & $\begin{array}{l}\text { - Mean: } 9.7 \\
\text { months** } \\
(2.9-20.4)\end{array}$ & $-N / A$ & $-N / A$ & $\begin{array}{l}\text { - 5-year risk: } \\
36 \% *\end{array}$ & $\begin{array}{l}\text { - Median: } 7 \\
\text { months* }\end{array}$ \\
\hline $\begin{array}{l}\text { - Progression or } \\
\text { recurrence stage/ } \\
\text { grade }\end{array}$ & $\begin{array}{l}-10 / 29 \\
(35 \%)^{* *} \\
\text { superficial or } \\
\text { CIS } \\
-5 / 29(17 \%) \\
\text { invasive or } \\
\text { metastasis }\end{array}$ & $-N / A$ & $-N / A$ & $-N / A$ & $-N / A$ & $\begin{array}{l}-4 / 20(20 \%)^{* *} \\
\text { (stages not } \\
\text { shown) }\end{array}$ & $\begin{array}{l}\text { - } 80 / 221 \text { to open } \\
\text { surgery* } \\
\text { (stages not } \\
\text { shown) }\end{array}$ \\
\hline $\begin{array}{l}\text { - Time to } \\
\text { progression }\end{array}$ & $-N / A$ & $-N / A$ & $-N / A$ & $-N / A$ & $-N / A$ & $-N / A$ & $\begin{array}{l}\text { - 5-year risk: } \\
40 \% *\end{array}$ \\
\hline $\begin{array}{l}\text { - Recurrence in } \\
\text { G2 }\end{array}$ & $\begin{array}{l}-40 / 120 \\
(33 \%)^{*}\end{array}$ & $\begin{array}{l}-11 / 36 \\
(30.6 \%)^{*}\end{array}$ & $\begin{array}{l}-56 / 141 \\
(40 \%)^{*}\end{array}$ & $-12 / 25(48 \%)^{*}$ & $-N / A$ & $-17 / 23(74 \%)^{*}$ & $\begin{array}{l}-566 / 686 \\
(82.5 \%)^{*}\end{array}$ \\
\hline $\begin{array}{l}\text { - Time to } \\
\text { recurrence } \\
\text { (range) }\end{array}$ & $-N / A$ & - NA & $\begin{array}{l}\text { - Mean: } 9.4 \\
\text { months** } \\
(2.4-43.4)\end{array}$ & $-N / A$ & $-N / A$ & $\begin{array}{l}\text { - 5-year risk: } \\
73 \% *\end{array}$ & $\begin{array}{l}\text { - Median: } 6 \\
\text { months* }\end{array}$ \\
\hline $\begin{array}{l}\text { - Progression or } \\
\text { recurrence stage/ } \\
\text { grade }\end{array}$ & $\begin{array}{l}-10 / 120 \\
(8 \%)^{* *} \\
\text { recurrence as } \\
\text { superficial/ } \\
\text { CIS } \\
-30 / 120 \\
(25 \%) \\
\text { recurrence } \\
\text { as invasive or } \\
\text { metastasis }\end{array}$ & $-N / A$ & $-N / A$ & $-N / A$ & $-N / A$ & $\begin{array}{l}-7 / 23(30 \%)^{* *} \\
\text { (stages not } \\
\text { shown) }\end{array}$ & $\begin{array}{l}\text { - 270/686 to open } \\
\text { surgery* } \\
\text { (stages not } \\
\text { shown) }\end{array}$ \\
\hline $\begin{array}{l}\text {-Time to } \\
\text { progression }\end{array}$ & $-N / A$ & $-N / A$ & $-N / A$ & $-N / A$ & $-N / A$ & $-N / A$ & $\begin{array}{l}\text { - 5-year risk: } \\
43 \% *\end{array}$ \\
\hline Statistical Tests: & $\begin{array}{l}\text { Chi square } \\
\text { test }\end{array}$ & $\begin{array}{l}\text { Fisher's exact } \\
\text { test }\end{array}$ & $\begin{array}{l}\text { Kaplan- } \\
\text { Meier/log- } \\
\text { rank test }\end{array}$ & $\begin{array}{l}\text { Chi-square } \\
\text { test }\end{array}$ & $-N / A$ & $\begin{array}{l}\text { Kaplan-Meier/ } \\
\text { log-rank test }\end{array}$ & $\begin{array}{l}\text { Kaplan-Meier/ } \\
\text { log-rank test }\end{array}$ \\
\hline$p$ value & $\begin{array}{l}{ }^{*} p=0.065 \\
{ }^{*} p<0.01\end{array}$ & $\begin{array}{l}{ }^{*} x^{2}=7.79 \\
{ }^{*} p<0.01\end{array}$ & $\begin{array}{l}p=0.28 \\
\text { (NS) }\end{array}$ & ${ }^{*} p<0.05$ & & $\begin{array}{l}{ }^{*} p=0.03 \\
{ }^{*} p=0.4 \text { (NS) }\end{array}$ & ${ }^{*} p=0.6$ (NS) \\
\hline $\begin{array}{l}\text { Univariate results } \\
\text { summary }\end{array}$ & $\begin{array}{l}\text { - NS } \\
\text { difference } \\
\text { in overall } \\
\text { recurrences } \\
\text { - Significantly } \\
\text { more } \\
\text { superficial } \\
\text { bladder } \\
\text { tumour } \\
\text { recurrence } \\
\text { in G1 }\end{array}$ & $\begin{array}{l}\text { - Significantly } \\
\text { more } \\
\text { recurrence } \\
\text { in G1 }\end{array}$ & $\begin{array}{l}-{ }^{*} N S \text { more } \\
\text { failure in G2 } \\
\text { - NS time to } \\
\text { failure }\end{array}$ & $\begin{array}{l}\text { - Significantly } \\
\text { more } \\
\text { recurrence } \\
\text { in G1 }\end{array}$ & $-N / A$ & $\begin{array}{l}\text { - Significantly } \\
\text { more recurrence } \\
\text { in G2 } \\
\text { - NS difference } \\
\text { in progression }\end{array}$ & $\begin{array}{l}\text { - NS difference } \\
\text { in median time } \\
\text { to recurrence or } \\
\text { progression in } 2 \\
\text { groups }\end{array}$ \\
\hline
\end{tabular}

Asterisks indicate data used for $p$ value calculations.

G: group; BCG: Bacille Calmette-Guérin; ASA: acetylsalicylic acid; CIS: carcinoma in situ; N/A: not available; NS: non-significant; HR: hazard ratio; ANOVA: analysis of variance. 


\begin{tabular}{|c|c|c|c|c|c|c|c|}
\hline & $\begin{array}{c}\text { Hudson et al. }{ }^{13} \\
1990\end{array}$ & $\begin{array}{c}\text { P'ng et al. }{ }^{15} \\
1993\end{array}$ & $\begin{array}{l}\text { Witjes et } \\
\text { al. }{ }^{16} 1993\end{array}$ & $\begin{array}{c}\text { Rogerson }^{14} \\
1994\end{array}$ & $\begin{array}{c}\text { Heiner et al. }{ }^{12} \\
2008\end{array}$ & Gee et al. ${ }^{11} 2008$ & $\begin{array}{c}\text { Boorjian et al. }{ }^{10} \\
2009\end{array}$ \\
\hline $\begin{array}{c}\text { G1/G2 } \\
\text { Medication }\end{array}$ & $\begin{array}{l}29 / 120 \\
\text { Combined }\end{array}$ & $\begin{array}{c}9 / 36 \\
\text { Combined }\end{array}$ & $\begin{array}{c}42 / 141 \\
\text { Combined }\end{array}$ & $\begin{array}{c}13 / 25 \\
\text { Combined }\end{array}$ & $\begin{array}{c}7 / 51 \\
\text { Warfarin }\end{array}$ & $\begin{array}{c}20 / 23 \\
\text { ASA }\end{array}$ & $\begin{array}{l}221 / 686 \\
\text { Combined }\end{array}$ \\
\hline \multicolumn{8}{|c|}{ Multivariate analyses results } \\
\hline - Recurrences & N/A & $\mathrm{N} / \mathrm{A}$ & $\mathrm{N} / \mathrm{A}$ & $\mathrm{N} / \mathrm{A}$ & $\begin{array}{l}\text { - Sig. } \\
\text { increased risks } \\
\text { in Gp. } 1 \\
\text { - Average rank } \\
2.6 \\
-p<0.01\end{array}$ & $\begin{array}{l}\text { - Significant } \\
\text { decreased risk } \\
\text { in G1 } \\
\text { - HR: } 0.179 \\
(0.062-0.516) p= \\
0.001\end{array}$ & $\mathrm{~N} / \mathrm{A}$ \\
\hline - Progression & $\mathrm{N} / \mathrm{A}$ & $\mathrm{N} / \mathrm{A}$ & $\mathrm{N} / \mathrm{A}$ & $\mathrm{N} / \mathrm{A}$ & $\mathrm{N} / \mathrm{A}$ & $\mathrm{N} / \mathrm{A}$ & $\mathrm{N} / \mathrm{A}$ \\
\hline $\begin{array}{l}\text { - Statistical tests } \\
\text { - Controlled } \\
\text { covariates }\end{array}$ & N/A & N/A & $\mathrm{N} / \mathrm{A}$ & $\mathrm{N} / \mathrm{A}$ & $\begin{array}{l}\text { - ANOVA } \\
\text { - Controlling } \\
\text { for age } \\
\text { and BCG } \\
\text { complications }\end{array}$ & $\begin{array}{l}\text { - Multivariate } \\
\text { analysis } \\
\text { - Age, sex, } \\
\text { smoking, } \\
\text { maintenance } \\
\text { BCG, CIS and } \\
\text { presence of } \\
\text { papillary tumour }\end{array}$ & $\mathrm{N} / \mathrm{A}$ \\
\hline
\end{tabular}

G: group; BCG: Bacille Calmette-Guérin; ASA: acetylsalicylic acid; CIS: carcinoma in situ; N/A: not available; NS: non-significant; HR: hazard ratio; ANOVA: analysis of variance.

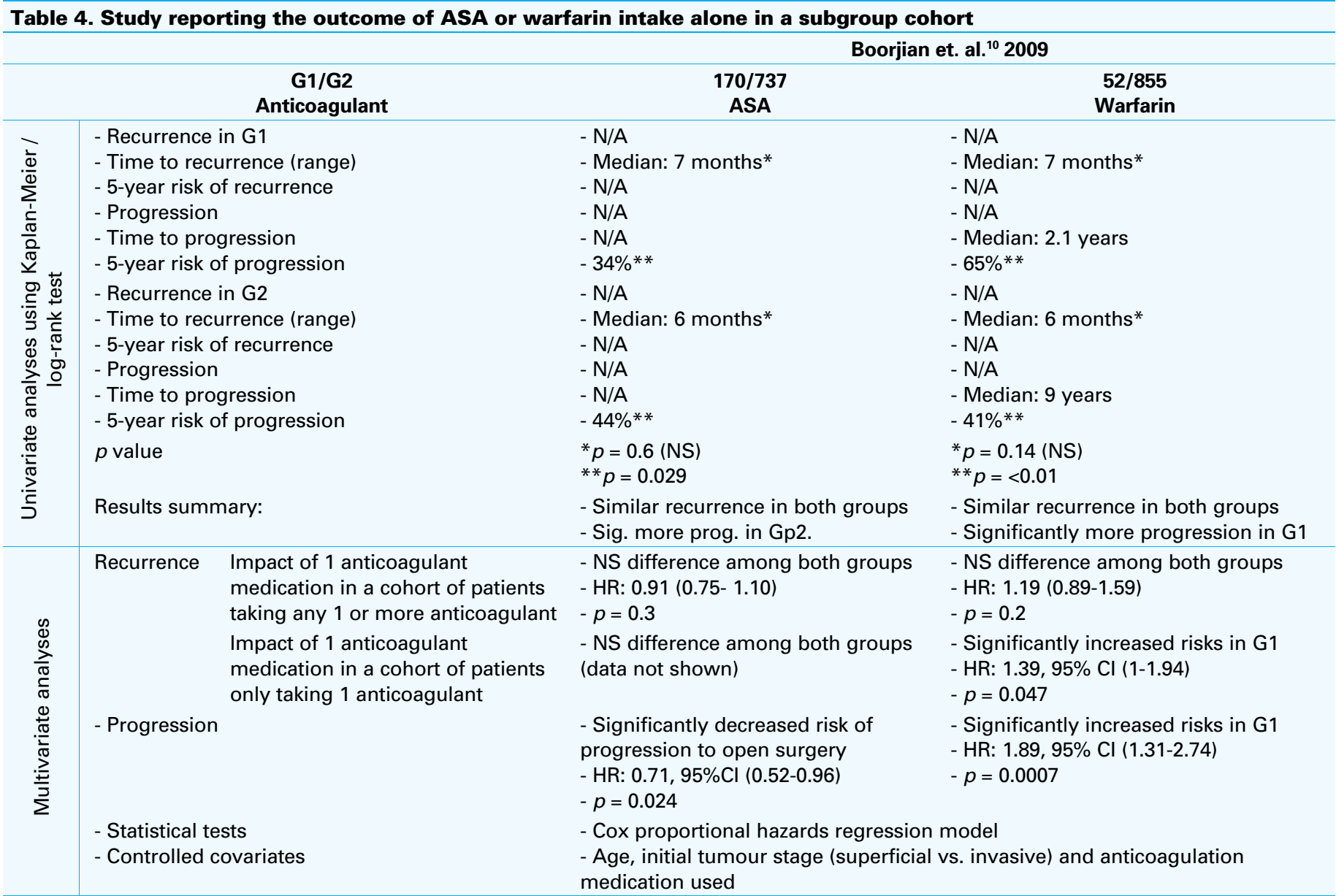

Asterisks indicate data used for $p$ value calculations. N/A: not available; NS: non-significant; G: group; HR: hazard ratio; Cl: confidence interval. 
on whether they were taking anticoagulant or antiplatelet medications or not, respectively.

The ideal method to test our outcomes is to design randomized, controlled prospective studies. However, if no randomized studies exist, observational studies showing consistent results can still be used to reasonably draw valid conclusion; there is a caveat: if such studies are retrospective and inadequately conducted, they are prone to inherent selection biases.

Given the heterogeneity of these studies we could not perform a meta-analysis. Reviewing the literature has revealed significant differences:

1. Failure to reveal critical baseline patient characteristics;

2. Limited number of study patients;

3. Exclusions of up to more than half of patients due to unavailability of medication records;

4. Different mean age among tested groups;

5. Different duration and follow-up schedules;

6. Different use of anticoagulant or antiplatelet medication type, dosage and duration;

7. Combining the outcome of different anticoagulant or antiplatelet medications groups whether taken separately or together;

8. Failure to take into account the concomitant intake of other medications;

9. Diverse risk factors for bladder cancer prognosis;

10. Different pre-treatment tumour stages and grades;

11. Variable BCG strains used along with multiple BCG regimens;

12. Unavailability of BCG complications;

13. Unknown recurrence stage and grade;

14. Different tested endpoints using variable statistical analyses; and

15. An ill-defined time to recurrence with possible introduction of lead time biases.

Despite all these differences, most investigators did not control for the vast variation in their examined variables. Among those studies that performed univariate analysis, 5 combined the different groups of anticoagulant or antiplatelet medications. ${ }^{10,13-16}$ Only 1 manuscript tested individual recurrences based on invasion stage and reported more superficial recurrences in group 1 patients. ${ }^{13}$ However, most publications only compared overall recurrences and $60 \%$ of the studies failed to show any statistical difference in disease recurrence among both groups. ${ }^{10,13,16}$ The conference abstract that was excluded from this study also failed to show any significant difference in recurrence-free survival rates following antiplatelet administration. ${ }^{9}$ Two studies examined tumour progression following BCG and both found no differences among both groups. ${ }^{10,13}$

Only 2 published manuscripts reported the outcome of patients taking oral warfarin alone. One of the studies used multivariate analyses in a cohort of patients taking 1 or more anticoagulant medications and did not find any significant difference in recurrence rates among both groups after controlling for the type of anticoagulant medication used. It was only when the authors sub-selected a cohort (using only 1 anticoagulant medication) and compared those to a group of controls that a significantly increased risk of recurrence following BCG was found among patients on warfarin (Table 4). ${ }^{10}$ Such analytical strategy raises serious concerns regarding the possibility of selection bias.

This manuscript had further limitations some of which were acknowledged by the authors, given the difficult nature of conducting and planning such studies. These limitations involved combining pTa, pT1 and CIS tumour stages that are known to have dissimilar risks and prognosis under one category. It also included the inclusion of a significant proportion of patients with unknown stage and grade. Furthermore authors did not control for other important risk factors, like smoking history and duration of warfarin medication use. Moreover these patients never received a maintenance intravesical BCG.

Similarly, another study compared the outcome of warfarin intake alone by ranking the response to BCG therapy into an ordinal scale of 1 to 4 and averaging those responses. ${ }^{12}$ The authors reported a poorer response rank of 2.6 following BCG in patients on warfarin independent of age. This study also had many limitations, including drawing conclusions from only 7 patients in the warfarin group and not controlling for all of the other risk factors. Furthermore, these scale numbers represent a rank with no numerical measurable differences between groups, thus computing means becomes very confusing and caution should be taken prior to drawing conclusion from such statistical analysis. Additionally, analysis of variance (ANOVA) for such categorical values may lead to misleading results that go beyond the normal chance of Type I and Type II errors. ${ }^{21}$

By means of multivariate analyses, 2 publications tested intravesical BCG outcome in patients only taking ASA and both described a seemingly protective effect. ${ }^{10,11}$ While 1 study reported significantly fewer recurrences with no difference in progression in patients taking ASA, ${ }^{11}$ the other manuscript failed to find any significant difference in recurrence and found a significantly decreased risk of progression to open surgery in the ASA group..$^{10}$ Several mechanisms have been suggested to elucidate the possible ASA beneficiary effects. ${ }^{10}$ It may be in part explained by the fact that local or systemic anticoagulants may prevent tumour cell adhesion and implantation to injured urothelium. ${ }^{22,23}$ Furthermore, ASA may exert its protective role through its non-selective cyclooxygenase (COX)-2 inhibition. COX-inhibiting drugs have antitumour activity in both canine and rodent models of urinary bladder cancer. ${ }^{24}$ Additionally COX-2 was found to be expressed in human CIS and invasive urothelial carcinoma, but not in the normal bladder. ${ }^{24}$ Moreover, COX 
inhibition in a murine model enhanced the production of interleukin- 12 by BCG activated dendritic cells and generated type $1 \mathrm{~T}$-helper response that may partially justify the improved BCG efficacy. ${ }^{25}$

The limitations of this study are similar to those in previously published studies. This study highlights the fact that our cumulative understanding in this area is very limited and insufficient to justify the practice of discontinuing warfarin prior to intravesical BCG therapy. We also suggest that patients continue their ASA medications for their cardiovascular benefits rather than for any other potential benefits in changing the prognosis of their bladder cancer.

\section{Conclusions}

A total of 7 published manuscripts examined the outcome of intravesical BCG in patients receiving anticoagulant or antiplatelet medications with contradictory results. Based on published data, there is no strong evidence to either encourage or discourage anticoagulant or antiplatelet medications while on intravesical BCG therapy yet. Further experimental and properly conducted clinical studies are still needed to further explore drug interactions and elucidate the mechanism of action of BCG.

Competing interests: Dr. Lazo-Langner has received honoraria for Advisory Board membership from Pfizer and LeoPharma. He has also received an unrestricted educational grant and honoraria from Alexion and Pfizer. He has participated in clinical trials for Pfizer, LeoPharma, Bayer and Daiichi-Sankyo. Dr. Fahmy, Ms. lansavichene and Dr. Pautler all declare no competing financial or personal interests.

This paper has been peer-reviewed.

\section{References}

1. Canadian Cancer Society's Steering Committee on Cancer Statistics: Canadian Cancer Statistics (2012). http://www.cancer.ca/ /media/cancer.ca/CW/cancer\%\%20information/cancer\%20101/Canadian\%20 cancer\%20statistics/Canadian-Cancer-Statistics-2012-English.pdf. Accessed 0ctober 29, 2013.

2. Wities JA. Management of BCG failures in superficial bladder cancer: a review. Eur Urol 2006;49:790-7. http://dx.doi.org/10.1016/i.eururo.2006.01.017

3. Zlotta AR, Fleshner NE, Jewett MA. The management of BCG failure in non-muscle-invasive bladder cancer: an update. Can Urol Assoc J 2009;3:S199-205.

4. Kavoussi LR, Brown EJ, Ritchey JK, et al. Fibronectin-mediated Calmette-Guerin bacillus attachment to murine bladder mucosa. Requirement for the expression of an antitumor response. I Clin Invest 1990;85:62-7. http://dx.doi.org/10.1172/JCI114434

5. Ratliff TL, Palmer JO, MCGarr JA, et al. Intravesical Bacillus Calmette-Guerin therapy for murine bladder tumors: initiation of the response by fibronectin-mediated attachment of Bacillus Calmette-Guerin. Cancer Res 1987;47:1762-6.
6. Hudson MA, Brown EJ, Ritchey JK, et al. Modulation of fibronectin-mediated Bacillus Calmette-Guerin attachment to murine bladder mucosa by drugs influencing the coagulation pathways. Cancer Res 1991;51:3726-32.

7. Shen ZJ, Wang Y, Ding GQ, et al. Study on enhancement of fibronectin-mediated bacillus Calmette-Guerin attachment to urinary bladder wall in rabbits. World J Urol 2007;25:525-9. http://dx.doi.org/10.1007/ s00345-007-0198-z

8. Moher D, Liberati A, Tetzlaff J, et al. Preferred reporting items for systematic reviews and metaanalyses: the PRISMA statement. J Clin Epidemiol 2009;62:1006-12. http://dx.doi.org/10.1016/i. jclinepi.2009.06.005

9. Yuge K, Kikuchi E, Tanaka N, et al. Clinical impact of anti-platelet medication in patients with non-muscle invasive bladder cancer treated with BCG therapy. Urology 2012;80:S218.

10. Boorijan SA, Berglund RK, Maschino AC, et al. Fibrin clot inhibitor medication and efficacy of bacillus Calmette-Guerin for bladder urothelial cancer. J Urol 2009;182:1306-12. http://dx.doi.org/10.1016/i. juro.2009.06.026

11. Gee JR, Jarrard DF, Bruskewitz RC, et al. Reduced bladder cancer recurrence rate with cardioprotective aspirin after intravesical bacille Calmette-Guerin. BJU Int 2009; 103:736-9. http://dx.doi.org/10.1111/ i.1464-410X.2008.08123.x

12. Heiner JG, Terris MK. Effect of advanced age on the development of complications from intravesical bacillus Calmette-Guerin therapy. Urol Oncol 2008;26:137-40. http://dx.doi.org/10.1016/i. urolonc.2007.04.005

13. Hudson MA, Yuan JJ, Catalona WJ, et al. Adverse impact of fibrin clot inhibitors on intravesical bacillus Calmette-Guerin therapy for superficial bladder tumors. J Urol 1990;144:1362-4.

14. Rogerson JW. Intravesical bacille Calmette-Guerin in the treatment of superficial transitional cell carcinoma of the bladder. Br J Urol 1994;73:655-8.

15. $P^{\prime} \mathrm{Ng} \mathrm{K} \mathrm{B}$, Walsh MD, Seymour GJ, et al. The adverse effect of fibrin-clot inhibiting drugs on intravesical bacillus Calmette-Guerin efficacy for superficial bladder cancer. Aust N Z I Surg 1993;63:127-30.

16. Witjes JA, vd Meijden AP, Doesburg W, et al. Influence of fibrin clot inhibitors on the efficacy of intravesical Bacillus Calmette-Guerin in the treatment of superficial bladder cancer. The Dutch Southeast Cooperative Urological Group. Eur Urol 1993;23:366-70.

17. Zuiverloon TC, Nieuweboer AJ, Vekony H, et al. Markers predicting response to bacillus Calmette-Guerin immunotherapy in high-risk bladder cancer patients: a systematic review. Eur Urol 2012;61:128-45. http://dx.doi.org/10.1016/i.eururo.2011.09.026

18. Alexandroff $A B$, Jackson $A M, O^{\prime}$ Donnell $M A$, et al. $B C G$ immunotherapy of bladder cancer: 20 years on. Lancet 1999;353:1689-94. http://dx.doi.org/10.1016/S0140-6736(98)07422-4

19. Hudson MA, Ritchey JK, Catalona WJ, et al. Comparison of the fibronectin-binding ability and antitumor efficacy of various mycobacteria. Cancer Res 1990;50:3843-7.

20. Pan CW, Shen ZJ, Ding GQ. The effect of intravesical instillation of antifibrinolytic agents on bacillus Calmette-Guerin treatment of superficial bladder cancer: a pilot study. J Urol 2008;179:1307-11. http:// dx.doi.org/10.1016/i.juro.2007.11.045

21. Jaeger TF. Categorical Data Analysis: Away from ANOVAs (transformation or not) and towards Logit Mixed Models. J Mem Lang 2008;59:434-46. http://dx.doi.org/10.1016/i.jml.2007.11.007

22. See WA, Chapman PH. Heparin prevention of tumor cell adherence and implantation on injured urothelial surfaces. J Urol 1987;138:182-6.

23. See WA, Miller JS, Williams RD. Pathophysiology of transitional tumor cell adherence to sites of urothelial injury in rats: mechanisms mediating intravesical recurrence due to implantation. Cancer Res 1989;49:5414-8.

24. Mohammed SI, Knapp DW, Bostwick DG, et al. Expression of cyclooxygenase-2 (COX-2) in human invasive transitional cell carcinoma (TCC) of the urinary bladder. Cancer Res 1999;59:5647-50.

25. Dovedi SJ, Kirby JA, Atkins H, et al. Cyclooxygenase-2 inhibition: a potential mechanism for increasing the efficacy of bacillus calmette-guerin immunotherapy for bladder cancer. J Urol 2005; 174:332-7. httr:// dx.doi.org/10.1097/01.ju.0000161589.85869.ae

Correspondence: Dr. Stephen E. Pautler, Associate Professor, Divisions of Urology \& Surgical Oncology, Departments of Surgery \& Oncology, Schulich School of Medicine \& Dentistry, Western University, South West Surgical Oncology Lead, Cancer Care Ontario, Urology, St. Joseph's Hospital, 268 Grosvenor St., London, ON N6A 4V2; fax: 519646 6037; stephen.pautler@sihc.london.on.ca 